\title{
Skewness of energy-loss straggling and multiple-scattering energy distributions
}

\author{
M. Mayer ${ }^{\mathrm{a}}, \mathrm{K}$. Arstila $^{\mathrm{b}}$ and U. von Toussaint ${ }^{\mathrm{a}}$ \\ ${ }^{a}$ Max-Planck-Institut für Plasmaphysik, EURATOM Association, Boltzmannstr. 2, \\ D-85748 Garching, Germany \\ b IMEC, Kapeldreef 75, 3001 Leuven, Belgium
}

\begin{abstract}
Energy spread distributions due to electronic energy-loss straggling and multiple small-angle scattering of swift ions in matter are approximated by asymmetric Gaussian functions consisting of two-piece normal distributions. These skewed energy distributions approximate the distributions more accurately than the conventional approximation by Gaussian functions. The results are compared to experimental data and Monte-Carlo simulations using the MCERD code and are in good agreement. The use of these skewed distributions allows to achieve a higher accuracy in the computer simulation of ion beam analysis energy spectra.
\end{abstract}

\section{Introduction}

The slowing down of swift ions in matter is always associated with energy spread due to energy-loss straggling [1] and multiple small-angle scattering [2-4]. The quantitative evaluation of measured particle energy spectra obtained by ion beam analysis (IBA) methods requires a precise computer simulation of these spectra, and several computer codes were developed for this purpose [5,6]. The most popular are SIMNRA [7,8], RUMP [9], and NDF [10]. All these codes approximate the energy spread distributions by Gaussian functions. However, as has already been shown experimentally for energy-loss straggling [11,12] and by Monte-Carlo simulations for multiple small-angle scattering [13], these energy spread distributions are usually of non-Gaussian shape and skewed.

An accurate calculation of the shape of electronic energy-loss straggling distributions is possible within the framework of a general stopping and straggling theory, such as the Bohr, Bethe, or Bloch theory [11,12,1]. This requires an additional numerical convolution, which increases the computing time by about 
one order of magnitude: This is impractical due to computing time restrictions in simulation codes, which are often used interactively. Another limitation is, that the shape of the straggling distribution cannot be calculated if empirical or semi-empirical stopping or straggling models (such as SRIM stopping powers [14] or Yang straggling [15]) are used, because these models provide only information about one specific moment of the energy distribution: A stopping power model gives the mean energy loss and mean energy, a straggling model the variance of the straggling. But it is usually impossible to get information about higher moments (such as the skewness and kurtosis) from such semiempirical models. Because these models often provide a better accuracy than a general stopping and straggling theory, such semi-empirical models are often used in practice.

The available analytical theory of multiple small-angle scattering [2-4] intrinsically assumes symmetry of the angular and energy spread distributions and does not provide information about their skew. Monte-Carlo simulation codes, such as MCERD [16] and CORTEO [17], allow a precise calculation of multiple scattering energy distributions, but these codes are slower by three to four orders of magnitude than the above mentioned single scattering codes.

The different energy spread distributions relevant for IBA (electronic energyloss straggling, geometrical straggling, multiple scattering, energy spread due to porosity) can be approximated with sufficient accuracy by two-piece normal distributions (TPNDs). This slows down calculations only marginally and provides energy distributions with correct mean value, variance and third moment, thus resulting in higher accuracy in spectrum simulation. From the different classes of skewed Gaussian-type functions (Gram-Charlier expansion with linear term [18, section 3.15.8], skew-normal distribution [19], and other), the TPND has been selected due to its good compatibility with the calculational schemes for IBA.

\section{The two-piece normal distribution (TPND)}

The two-piece normal distribution (TPND) (sometimes called binormal distribution or joined half-Gaussian) $f(x)$ is defined by

$$
f(x)=\left\{\begin{array}{l}
A \exp \left(-\frac{\left(x-x_{0}\right)^{2}}{2 \sigma_{1}^{2}}\right) \text { if } x \leq x_{0} \\
A \exp \left(-\frac{\left(x-x_{0}\right)^{2}}{2 \sigma_{2}^{2}}\right) \text { if } x>x_{0}
\end{array}\right.
$$

$x_{0}$ is the mode of the distribution, $\sigma_{1}$ and $\sigma_{2}$ are the standard deviations towards lower and higher values. $A$ is a normalization factor. The half widths 
at half maximum (HWHMs) towards lower and higher values, $h_{1}$ and $h_{2}$, are connected to the corresponding standard deviations through

$$
h_{i}=\sqrt{2 \ln 2} \sigma_{i}
$$

where $i$ is either 1 or 2 .

The moments of a TPND can be found for example in [20]. The mean value $F=\int f(x) x d x$ is given by

$$
F=x_{0}+\sqrt{\frac{2}{\pi}}\left(\sigma_{2}-\sigma_{1}\right) .
$$

The variance $V=\int f(x)(x-F)^{2} d x$ is

$$
V=\sigma_{1} \sigma_{2}+\left(1-\frac{2}{\pi}\right)\left(\sigma_{2}-\sigma_{1}\right)^{2}
$$

The third central moment $M=\int f(x)(x-F)^{3} d x$ is

$$
\begin{aligned}
M & =\sqrt{\frac{2}{\pi}} \sigma_{1} \sigma_{2}\left(\sigma_{2}-\sigma_{1}\right)+\sqrt{\frac{2}{\pi}}\left(\frac{4}{\pi}-1\right)\left(\sigma_{2}-\sigma_{1}\right)^{3} \\
& \approx \sqrt{\frac{2}{\pi}} \sigma_{1} \sigma_{2}\left(\sigma_{2}-\sigma_{1}\right) \quad \text { for } \sigma_{1} \approx \sigma_{2} .
\end{aligned}
$$

For not too strongly skewed distributions, i.e. if the difference between $\sigma_{1}$ and $\sigma_{2}$ is below about $30 \%, x_{0}, \sigma_{1}$ and $\sigma_{2}$ can be calculated analytically for given $F, V$ and $M$ :

$$
\begin{aligned}
& \sigma_{1}=-\frac{\beta}{2}+\sqrt{\frac{\beta^{2}}{4}-a \beta^{2}+V} \\
& \sigma_{2}=\frac{\beta}{2}+\sqrt{\frac{\beta^{2}}{4}-a \beta^{2}+V} \\
& x_{0}=F-\frac{M}{V}
\end{aligned}
$$

with the abbreviations

$$
a=1-\frac{2}{\pi} \approx 0.3634
$$


and

$$
\beta=\sqrt{\frac{\pi}{2}} \frac{M}{V} .
$$

Eqs. 7 and 8 are deduced by using eq. 6 instead of eq. 5 . For $\sigma_{2}=(1+\delta) \sigma_{1}$ a term of the order $1 / 3 \delta^{3}$ is neglected.

The convolution of two TPNDs is, in general, not a TPND. The mean value $F$, variance $V$ and third moment $M$ are additive for a convolution. The convolution of a TPND $f_{1}$ with moments $F_{1}, V_{1}$ and $M_{1}$ and a TPND $f_{2}$ with moments $F_{2}, V_{2}$ and $M_{2}$ results in a function $f$ with moments

$$
\begin{aligned}
F & =F_{1}+F_{2} \\
V & =V_{1}+V_{2} \\
M & =M_{1}+M_{2} .
\end{aligned}
$$

For not too large asymmetries (with the difference between $\sigma_{1}$ and $\sigma_{2}$ being less than about $50 \%$ ), the convolution of two TPNDs is very close to a TPND, and $f$ can be approximated by a TPND with moments $F, V$ and $M$. The parameters of this TPND are then given by eqs. 7,8 and 9 .

\section{Asymmetry parameter of arbitrary distributions}

A possible measure of the skew of a distribution is its third standardized moment, the skewness $\gamma=M / V^{3 / 2}$. However, the skewness can be derived only with large errors from experimental data or from data obtained by MonteCarlo computer simulations due to the large influence of small fluctuations in the wings of the distributions. Some textbooks generally discourage to use the skewness for experimental data [21]. Moreover, angular and energy distributions due to multiple small-angle scattering have infinite variance and an undefined third moment [2], so that the skew of these distributions cannot be measured by $\gamma$. The problem of infinite variance was overcome in [2] by using the full width at half maximum (FWHM) instead of using the variance $V$. Following this way, we introduce the asymmetry parameter $\alpha$ as a measure of the skew of a distribution, with

$$
\alpha=\frac{h_{2}-h_{1}}{h_{1}+h_{2}} .
$$


$h_{2}$ is the half width at half maximum (HWHM) towards higher values, i.e. the distance between the value at which the distribution has decreased to its half maximum and the mode. $h_{1}$ is the HWHM towards low values. $\alpha$ can be determined for any unimodal distribution, even if $V$ or $M$ are infinite or undefined, and can be determined with sufficient accuracy for experimental or Monte-Carlo data. Consequently we use the FWHM instead of the variance and the asymmetry parameter $\alpha$ instead of the skewness $\gamma$ for all comparisons to experimental or Monte-Carlo data.

\section{Computer simulations}

SIMNRA is a simulation program for RBS, ERD and NRA energy spectra [7,8,22]. Version 6.40 was used. SRIM 2003 stopping powers [14] were used for comparison to experimental data, ZBL electronic stopping powers [23] were used for comparison to MCERD.

MCERD is a TRIM-like Monte Carlo (MC) code in binary collision approximation [16,24]. ZBL electronic stopping powers [23] and the universal interaction potential were used. Electronic energy loss straggling was switched off.

\section{$5 \quad$ Electronic energy-loss straggling}

For small energy losses the energy distributions of slowing-down ions in matter are non-Gaussian with long tails towards low energies [25]. For larger energy losses the increasing number of statistically independent collisions tends to draw the energy distributions near to a Gaussian distribution. The energy distributions are typically almost Gaussian for energy losses in the range of 10-30\% of the primary energy, and their widths can be described by the Bohr theory [26], but have to be corrected for electron binding [27] and chargestate fluctuations [15] in order to achieve a sufficient accuracy. For energy losses exceeding about 30\% of the primary energy the widths and shapes of the energy distributions are determined by non-stochastic broadening or squeezing due to the shape of the stopping power [11,12,28,29].

As was already shown in [11], the first three moments are sufficient for an accurate approximation of the energy-loss distributions if the energy-loss is not too small. We obtain the mean value from the energy loss due to the stopping power $[30,31,23,32,14]$ and the variance from a straggling theory, such as [15]. As third moment we use nonrelativistic free Coulomb scattering [1, chapter 8.11]. Propagation of the HWHM's in thick layers is taken approximately into account by calculating the energy losses of the mode energy $E_{0}$ and the 
energies $E_{0}-h_{1}$ and $E_{0}+h_{2}$ during a step $\Delta x$. No information is available about the third moment of charge state fluctuations [15]: These are assumed to be Gaussian.

The $\Gamma$-distribution was proposed in [33] for modeling the shape of the energyloss distribution. However, because the $\Gamma$-distribution has only two adjustable parameters (mean value and variance), the third moment cannot be adjusted and will usually not coincide with the correct third moment of the distribution: The method proposed in [33] therefore generally will not approximate correctly the energy-loss distribution.

The straggling of $19.68 \mathrm{MeV}$ protons in aluminum is shown in Fig. 1. The experimental data were digitized from [29, Fig. 3], their half widths and asymmetry parameters were obtained by fitting. At this high incident energy multiple scattering is negligible, so that the shape of the energy distributions is determined by electronic energy-loss straggling only. The incident beam energy spread was $40 \mathrm{keV}$ FWHM [29] and is assumed to be Gaussian. The calculated half-widths of the energy distributions are in excellent agreement with the measurements. Bohr straggling describes the half-widths accurately at energies above about $15.5 \mathrm{MeV}$, i.e. for energy losses below about $20 \%$ of the primary energy. At larger energy losses the half-widths are larger than Bohr straggling due to straggling propagation. The asymmetry parameter, as shown in Fig. 1 bottom, is also in very good agreement with the experimental values. At very large energy losses (in depths above about $0.5 \mathrm{~g} / \mathrm{cm}^{2}$ ) the model somewhat underestimates the asymmetry.

The measured energy distributions of $19.68 \mathrm{MeV}$ protons after traversing $0.2675 \mathrm{~g} / \mathrm{cm}^{2}$ and $0.497 \mathrm{~g} / \mathrm{cm}^{2}$ aluminum are shown in Fig. 2. The energy distributions are well approximated by the TPNDs. The approximation by Gaussian energy distributions gives the correct widths (FWHMs) of the distributions, but the shape is not as well reproduced.

\section{Multiple scattering}

Szilágyi et al. [2] and Amsel et al. [4] have developed an analytical theory of multiple small angle scattering, which has been implemented in the computer codes DEPTH [2] and SIMNRA [7,13]. This analytical theory intrinsically assumes symmetry of all angular and energy spread distributions, which are approximated by (symmetric) Pearson VII distributions. The theory also neglects energy transfer to target atoms, i.e. nuclear straggling [13]. As has been already shown by comparison to Monte-Carlo simulation calculations [13], this theory correctly predicts the shape and FWHM of angular spread distributions: These are (almost) symmetric until large energy losses and can be 
described with sufficient accuracy by Pearson VII distributions. This theory also predicts the FWHM of energy spread distributions due to multiple scattering with sufficient accuracy [13]. It, however, does not describe correctly the shape of energy spread distributions: These are not symmetric, but always skewed towards low energies [13]. This skewness has two main reasons: Path length differences of particles scattered to different sides and nuclear straggling.

The influence of path length differences is shown schematically in Fig. 3: At oblique incidence particles scattered to one side (trajectory A) on average have a shorter total path length (and corresponding energy loss) until they reach a specific depth $d$ than particles scattered to the other side (trajectory $\mathrm{B}$ ). The path length $l_{A}$ for trajectory $\mathrm{A}$ is given by $l_{A}=d / \cos (\alpha-\Delta \epsilon / 2)$, with $\alpha$ the angle of incidence and $\Delta \epsilon$ the chord angle (see Fig. 3 ). The path length $l_{B}$ for trajectory $\mathrm{B}$ is given by $l_{B}=d / \cos (\alpha+\Delta \epsilon / 2)$. For identical chord angles to both sides this results in an asymmetric distribution of path lengths and a corresponding asymmetric energy distribution. Path length differences play especially a role at grazing incidence and for heavy target elements.

Multiple scattering of $2 \mathrm{MeV}{ }^{4} \mathrm{He}$ in $\mathrm{Au}$ at $60^{\circ}$ angle of incidence, as calculated with the Monte-Carlo code MCERD and by SIMNRA, is shown in Fig. 4. The widths of the angular distributions (Fig. 4 top) are in excellent agreement between both codes. The widths of the energy spread distributions (Fig. 4 middle) are in excellent agreement until a depth of about $500 \mathrm{~nm}$. At larger depths SIMNRA overestimates the width. The asymmetry parameter $\alpha$ (Fig. 4 middle, see eq. 13 for the definition of $\alpha$ ) is in agreement between MCERD and SIMNRA within the error bars. The large error bars for the MCERD data are mainly due to the fit inaccuracy for the position of the mode of the energy distributions, which has a large influence on the determination of $\alpha$. The calculated energy distributions in depths of $100 \mathrm{~nm}, 300 \mathrm{~nm}$ and $500 \mathrm{~nm}$ are shown in Fig 5. At small energy losses $(100 \mathrm{~nm})$ the distribution is almost symmetric with tails towards lower and higher energies. These tails are characteristic for multiple scattering [4] and cannot be described by Gaussian-type functions. At larger energy losses (300 $\mathrm{nm}$ and $500 \mathrm{~nm}$ ) the distributions get more and more asymmetric. The high-energy parts of the distributions approach a Gaussian distribution, while the low-energy parts develop broad tails extending to very low energies. The body of these distributions can be well described by a TPND, which gives a better approximation to the Monte-Carlo results than a Gaussian. The low-energy tail is mainly due to energy transfer to target atoms, i.e. nuclear straggling. Energy transfer to target atoms is neglected in the Szilágyi/Amsel theory of multiple scattering [2,4], and nuclear straggling at moderate energy losses is so strongly skewed that it cannot be described by a skewed Gaussian-type function. A successful description of this low-energy tail therefore would require an additional convolution, which is very costly in terms of computing time. This is especially important for heavy ions, where 
the nuclear energy-loss straggling contribution may be large and cannot be approximated by any skewed Gaussian type of function.

\section{Conclusions}

The energy spread distributions due to electronic energy-loss straggling and multiple small-angle scattering are approximated by asymmetric Gaussian functions consisting of two-piece normal distributions. These skewed energy distribution have been implemented in the SIMNRA code version 6.40 and higher. The results are compared to existing experimental data and MonteCarlo simulations using the MCERD code and are in good agreement for light ions, such as protons and helium ions. The application of these skewed distributions offers an improved accuracy in spectrum simulation.

\section{References}

[1] P. Sigmund. Particle Penetration and Radiation Effects, vol. 151 of Springer Series in Solid State Sciences. Springer, Berlin, Heidelberg, 2006.

[2] E. Szilágyi, F. Pászti, and G. Amsel. Nucl. Instr. Meth. B 100 (1995) 103.

[3] E. Szilágyi. Nucl. Instr. Meth. B 161-163 (2000) 37.

[4] G. Amsel, G. Battistig, and A. L'Hoir. Nucl. Instr. Meth. B 201 (2003) 325.

[5] E. Rauhala, N.P. Barradas, S. Fazinic, M. Mayer, E. Szilágyi, and M. Thompson. Nucl. Instr. Meth. B 244 (2006) 436.

[6] N.P. Barradas, K. Arstila, G. Battistig, M. Bianconi, N. Dytlewski, C. Jeynes, E. Kótai, G. Lulli, M. Mayer, E. Rauhala, E. Szilágyi, and M. Thompson. Nucl. Instr. Meth. B 262 (2007) 281.

[7] M. Mayer. SIMNRA user's guide. Tech. Rep. IPP 9/113, Max-Planck-Institut für Plasmaphysik, Garching, 1997.

[8] M. Mayer. SIMNRA, a simulation program for the analysis of NRA, RBS and ERDA. In Proceedings of the 15th International Conference on the Application of Accelerators in Research and Industry (Woodbury, New York, 1999), J. L. Duggan and I. Morgan, Eds., vol. 475 of AIP Conference Proceedings, American Institute of Physics, p. 541.

[9] R. Doolittle. Nucl. Instr. Meth. B 9 (1985) 344.

[10] N.P. Barradas. J. Phys. D: Appl. Phys. 34 (2001) 2109.

[11] C. Tschalär. Nucl. Instr. Meth. 61 (1968) 141. 
[12] C. Tschalär. Nucl. Instr. Meth. 64 (1968) 237.

[13] M. Mayer, K. Arstila, K. Nordlund, E. Edelmann, and J. Keinonen. Nucl. Instr. Meth. B 249 (2006) 823.

[14] J.F. Ziegler. Nucl. Instr. Meth. B 219-220 (2004) 1027.

[15] Q. Yang, D.J. O'Connor, and Z. Wang. Nucl. Instr. Meth. B 61 (1991) 149.

[16] K. Arstila, T. Sajavaara, and J. Keinonen. Nucl. Instr. Meth. B 174 (2001) 163.

[17] François Schiettekatte. Nucl. Instr. Meth. B 266 (2008) 1880.

[18] B.R. Frieden. Probability, Statistical Optics, and Data Testing: A Problem Solving Approach, vol. 10 of Springer Series in Information Sciences. Springer, Berlin, 1991.

[19] A. Azzalini. Scandinavian Journal of Statistics 12 (1985) 171.

[20] J.S. Garvin and S.I. McClean. The Statistician 46 (1997) 33.

[21] W.H. Press, B.P. Flannery, S.A. Teukolsky, and W.T. Vetterling. Numerical Recipes. Cambridge University Press, Cambridge, New York, 1988.

[22] M. Mayer. SIMNRA: Simulation of RBS, ERD and NRA spectra. http://www.rzg.mpg.de/ mam/.

[23] J.F. Ziegler, J.P. Biersack, and U. Littmark. The Stopping and Range of Ions in Solids, vol. 1 of The Stopping and Ranges of Ions in Matter. Pergamon Press, New York, 1985.

[24] K. Arstila, J.A. Knapp, K. Nordlund, and B.L. Doyle. Nucl. Instr. Meth. B 219-220 (2004) 1058.

[25] P.V. Vavilov. Soviet Physics J.E.T.P. 5 (1957) 749.

[26] N. Bohr. Mat. Fys. Medd. Dan. Vid. Selsk. 18, 8 (1948) .

[27] W.K. Chu. Phys. Rev. 13 (1976) 2057.

[28] M.G. Payne. Phys. Rev. 185, 2 (1969) 611.

[29] C. Tschalär and H.D. Maccabee. Phys. Rev. B1 (1970) 2863.

[30] H.H. Andersen and J.F. Ziegler. Hydrogen - Stopping Powers and Ranges in All Elements, vol. 3 of The Stopping and Ranges of Ions in Matter. Pergamon Press, New York, 1977.

[31] J.F. Ziegler. Stopping Cross-Sections for Energetic Ions in all Elements, vol. 5 of The Stopping and Ranges of Ions in Matter. Pergamon Press, New York, 1980.

[32] G. Konac, S. Kalbitzer, Ch. Klatt, D. Niemann, and R. Stoll. Nucl. Instr. Meth. B 136-138 (1998) 159.

[33] N.P. Barradas, R.P. Pezzi, and I.J.R. Baumvol. Nucl. Instr. Meth. B 261 (2007) 422. 


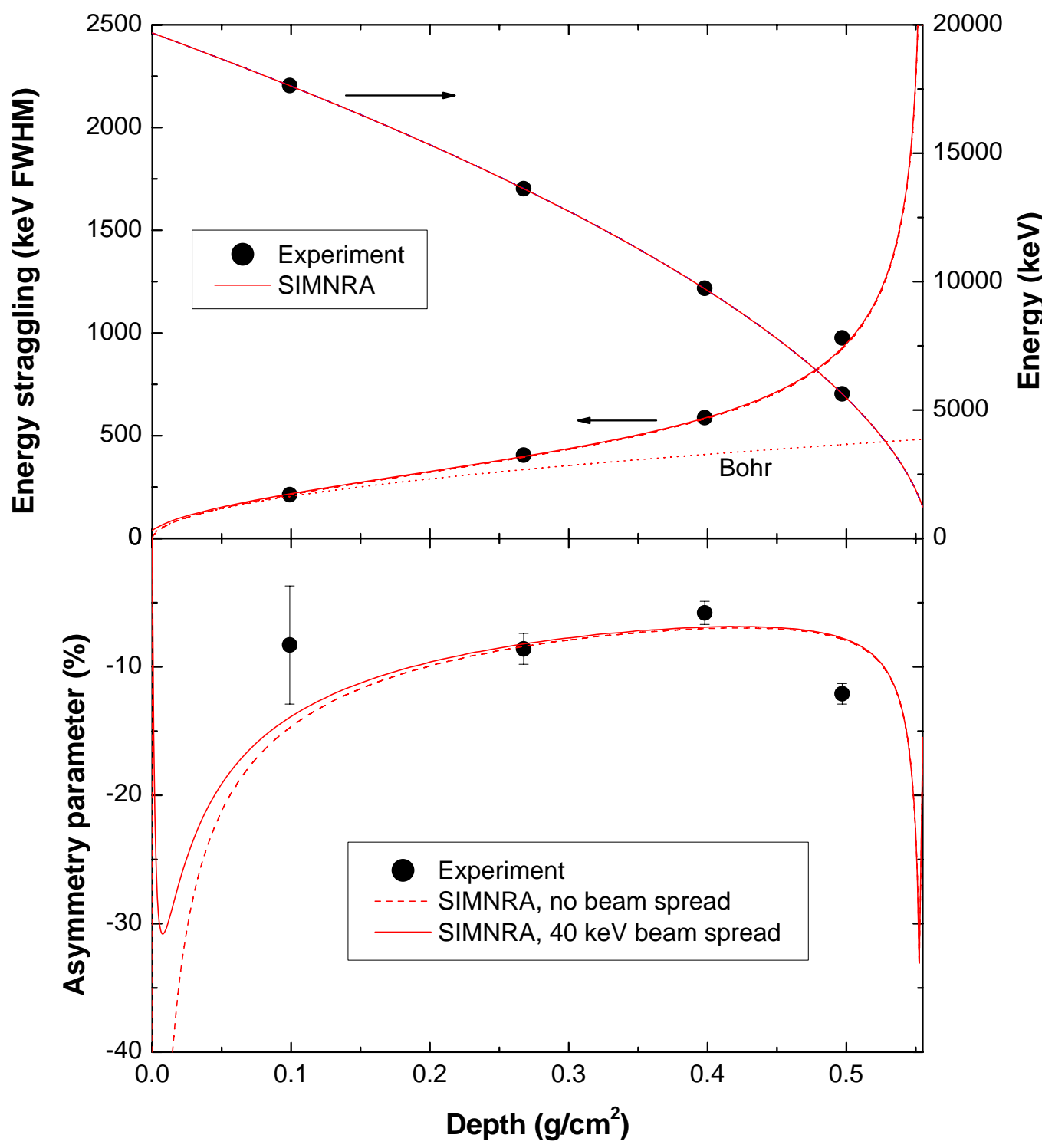

Fig. 1. Straggling of $19.68 \mathrm{MeV}$ protons in aluminum. Top: Mode energy and width (FWHM) of the straggling distributions. Bottom: Asymmetry parameter (see eq. 13) of the straggling distributions. Experimental data from [29]. Solid lines: $40 \mathrm{keV}$ Gaussian energy spread of incident beam; Dashed lines: Without energy spread of incident beam. 


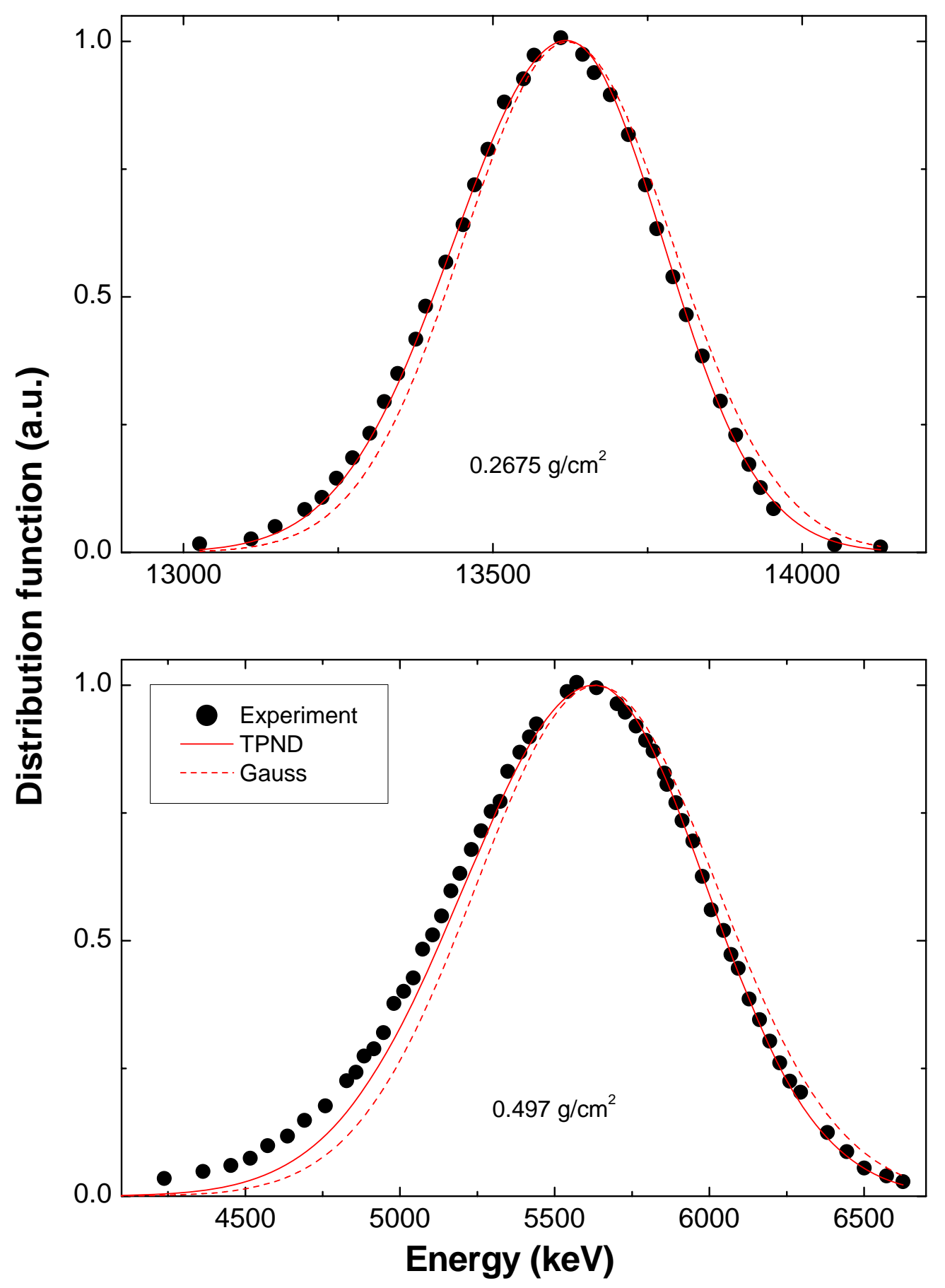

Fig. 2. Energy distributions of $19.68 \mathrm{MeV}$ protons after traversing $0.2675 \mathrm{~g} / \mathrm{cm}^{2}$ and $0.497 \mathrm{~g} / \mathrm{cm}^{2}$ aluminum. Dots: Experimental data from [29]; Solid line: SIMNRA calculation using a two-piece normal distribution; Dashed line: SIMNRA calculation using a Gaussian energy distribution. 


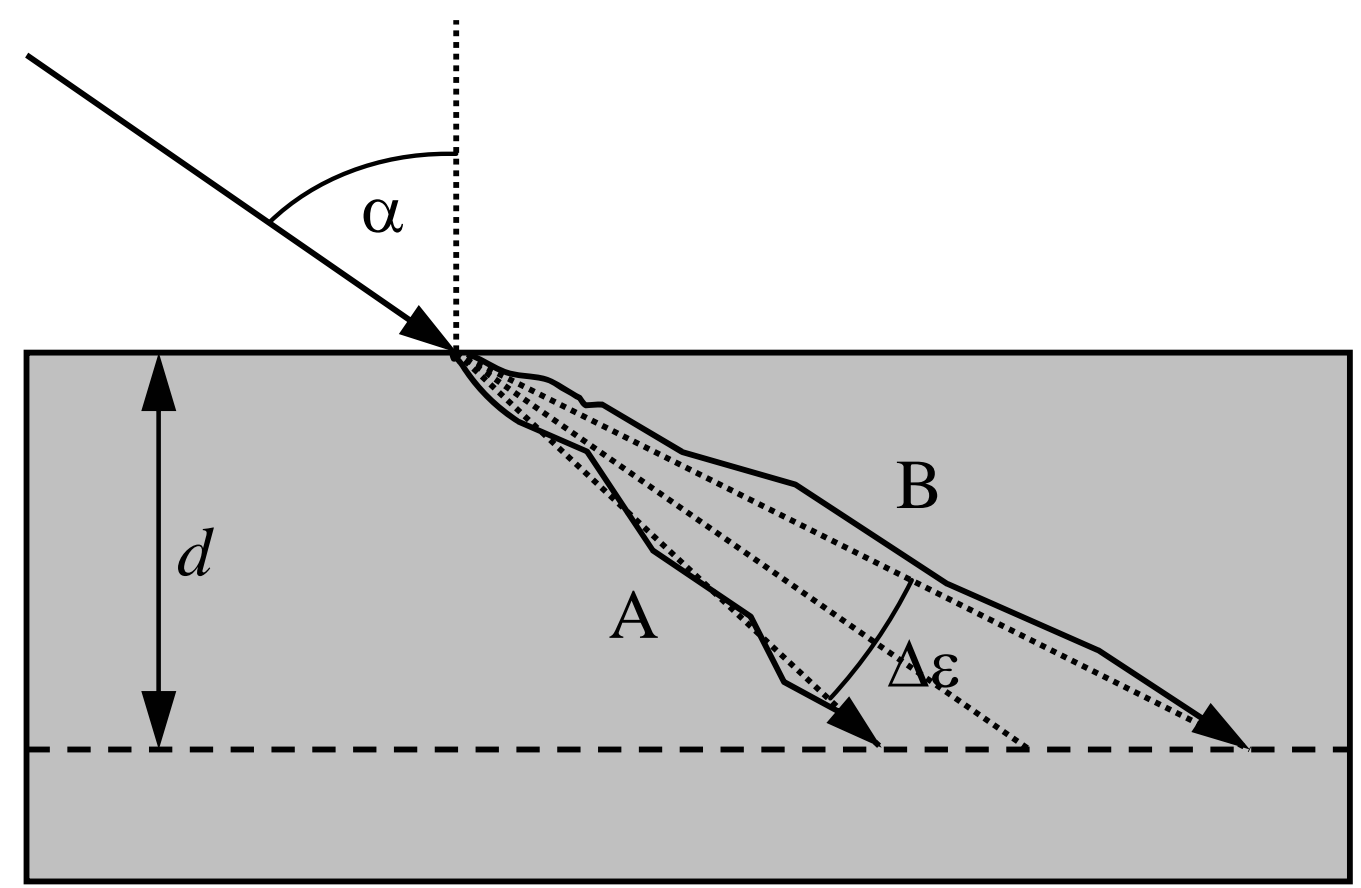

Fig. 3. Schematic representation of multiple scattering at oblique incidence. Depth $d$ and angle of incidence $\alpha$. Trajectories A and B are assumed to have chord angles of $\mp \epsilon / 2$, with the FWHM of the chord angle distribution $\Delta \epsilon$. 


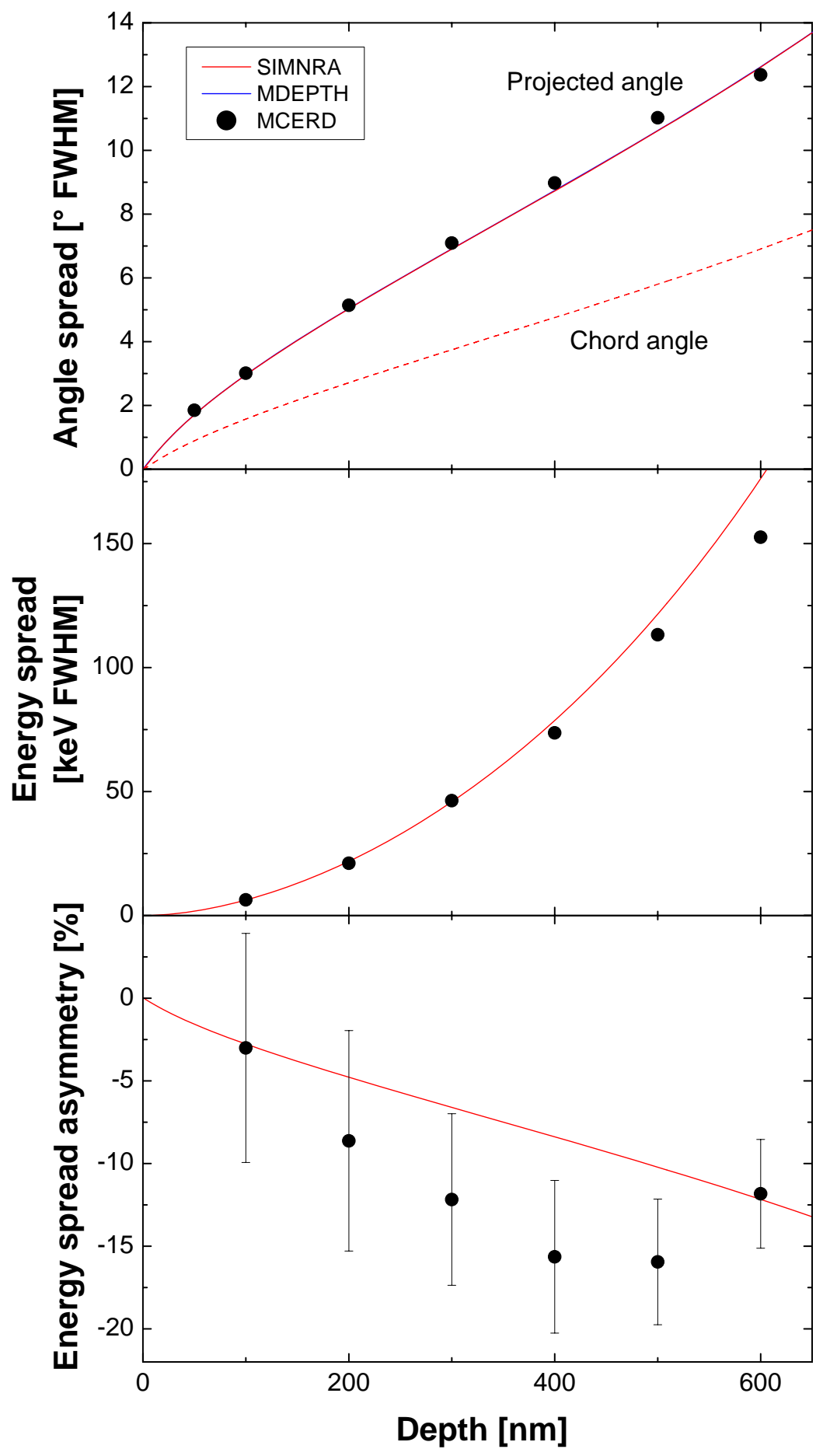

Fig. 4. Multiple scattering of $2 \mathrm{MeV}^{4} \mathrm{He}$ in gold at $60^{\circ}$ angle of incidence. Top: FWHMs of the angular spread distribution $\Delta \Phi$ and the chord angle distribution $\Delta \epsilon$; middle: FWHM of the energy spread distribution; bottom: Asymmetry parameter $\alpha$ of the energy spread distribution. Solid dots: Calculation with the Monte-Carlo code MCERD; lines: Calculation with SIMNRA. The MDEPTH calculation for the angular spread distribution coincides with the SIMNRA result. 


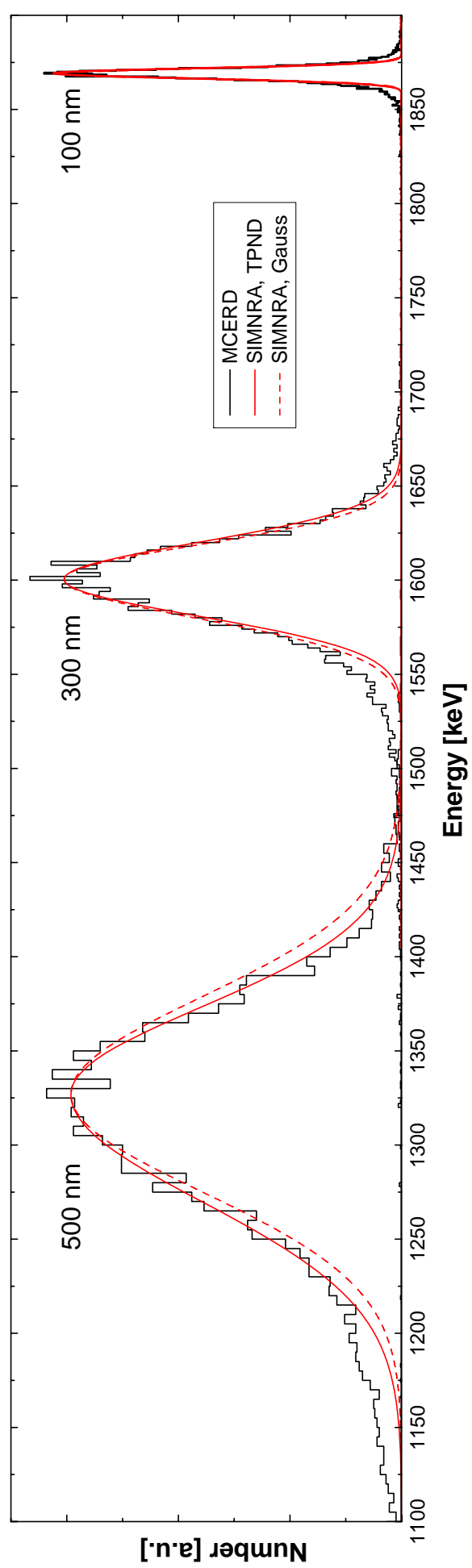

Fig. 5. Energy distributions due to multiple scattering of $2 \mathrm{MeV}{ }^{4} \mathrm{He}$ in gold at $60^{\circ}$ angle of incidence. in depths of $100 \mathrm{~nm}, 300 \mathrm{~nm}$ and $500 \mathrm{~nm}$. Histograms: Calculation with the Monte-Carlo code MCERD; dashed lines: Calculation with SIMNRA assuming a Gaussian shape of the distributions; solid lines: Calculation with SIMNRA assuming a two-piece normal distribution (TPND) shape of the distributions. 